\title{
ON THE INDEX OF SEQUENCES OVER CYCLIC GROUPS
}

\author{
WEIDONG GAO, YUANLIN LI, JIANGTAO PENG, CHRIS PLYLEY, AND GUOQING WANG
}

\begin{abstract}
Let $G$ be a finite cyclic group of order $n \geq 2$. Every sequence $S$ over $G$ can be written in the form $S=\left(n_{1} g\right) \cdot \ldots \cdot\left(n_{l} g\right)$ where $g \in G$ and $n_{1}, \ldots, n_{l} \in[1, \operatorname{ord}(g)]$, and the index $\operatorname{ind}(S)$ of $S$ is defined as the minimum of $\left(n_{1}+\ldots+n_{l}\right) / \operatorname{ord}(g)$ over all $g \in G$ with $\operatorname{ord}(g)=n$. In this paper we prove that a sequence $S$ over $G$ of length $|S|=n$ having an element with multiplicity at least $\frac{n}{2}$ has a subsequence $T$ with $\operatorname{ind}(T)=1$, and if the group order $n$ is a prime, then the assumption on the multiplicity can be relaxed to $\frac{n-2}{10}$. On the other hand, if $n=4 k+2$ with $k \geq 5$, we provide an example of a sequence $S$ having length $|S|>n$ and an element with multiplicity $\frac{n}{2}-1$ which has no subsequence $T$ with ind $(T)=1$. This disproves a conjecture given twenty years ago by Lemke and Kleitman.
\end{abstract}

\section{Introduction and Main Results}

Let $G$ be an additively written, finite cyclic group and $g \in G$ with $\operatorname{ord}(g)=|G|$. For a sequence

$$
S=\left(n_{1} g\right) \cdot \ldots \cdot\left(n_{l} g\right) \quad \text { over } G, \quad \text { where } l \in \mathbb{N}_{0} \quad \text { and } \quad n_{1}, \ldots, n_{l} \in[1, n],
$$

we set

$$
\|S\|_{g}=\frac{n_{1}+\ldots+n_{l}}{n}
$$

and then

$$
\operatorname{ind}(S)=\min \left\{\|S\|_{h} \mid h \in G \text { with } \operatorname{ord}(h)=|G|\right\} \in \mathbb{Q}_{\geq 0}
$$

denotes the index of $S$. The index of a sequence is a crucial invariant in the investigation of (minimal) zero-sum sequences (resp. of zero-sum free sequences) over cyclic groups. It was first addressed by Lemke and Kleitman ([11]), used as key tool by Geroldinger ([6․, page 736]), and then investigated by Gao [3] in a systematical way. Since then it has found a lot of attention in recent years (see [1, 2, 5, 8, 12, 13, 14, 15, 16]). We briefly discuss some key results.

If $S$ is a minimal zero-sum sequence, then $|S| \leq 3$, as well as $|S| \geq\left\lfloor\frac{n}{2}\right\rfloor+2$, implies that $\operatorname{ind}(S)=1$ (see [1], [14, [16]). In contrast to that, it was shown that for every $k \in\left[5,\left\lfloor\frac{n}{2}\right\rfloor+1\right]$, there is a minimal zero-sum subsequence $T$ of length $|T|=k$ and with $\operatorname{ind}(T) \geq 2$, and that the same is true for $k=4$ and $\operatorname{gcd}(n, 6) \neq 1$. This lead to the conjecture that, in case $\operatorname{gcd}(n, 6)=1$, every minimal zero-sum sequence $S$ over $G$ of length $|S|=4$ has $\operatorname{ind}(S)=1$. Li, Plyley, Yuan and Zeng [12] recently proved that this holds true if $n$ is a prime power, but the general case is still open.

In 1989, Lemke and Kleitman stated the following conjecture ([11, page 344]), which we formulate in the present language.

2010 Mathematics Subject Classification. 11B30, 11B50, $20 \mathrm{~K} 01$.

Key words and phrases. zero-sum sequences, index of sequences, cyclic groups. 
Conjecture 1.1. Let $G$ be a cyclic group of order $n, d$ a divisor of $n$, and let $S$ be a sequence over $G$ of length $|S|=n$. Then there exists a subsequence $T$ of $S$ and element $g \in G$ with $\operatorname{ord}(g)=n$ such that

$$
d\left|n\|T\|_{g}\right| n \text {. }
$$

In the special case $d=n$, this is equivalent to the existence of a subsequence $T$ with $\operatorname{ind}(T)=1$.

Indeed the above is the third of three interesting conjectures stated by Lemke and Kleitman in [11]. Their first conjecture has turned out to be true for all finite abelian groups (see [7]), and the second one is still open. In this paper we demonstrate that the above conjecture fails in general (see Theorem 1.2), but that it holds true under an additional assumption on the highest multiplicity of an element occurring in the sequence. Here are the main results of the present paper (for any undefined terminology or notation the reader is referred to the beginning of Section 2).

Theorem 1.2. Let $G$ be a cyclic group of order $n \geq 2$, where $n=4 k+2$ for some $k \geq 5$, and let $g \in G$ with $\operatorname{ord}(g)=n$. Then the sequence

$$
S=g^{\frac{n}{2}-3}\left(\frac{n}{2} g\right)\left(\left(\frac{n}{2}+1\right) g\right)^{\frac{n}{2}-1}\left(\left(\frac{n}{2}+2\right) g\right)^{\left\lfloor\frac{n}{4}\right\rfloor-2}
$$

has no subsequence $T$ with $\operatorname{ind}(T)=1$.

Theorem 1.3. Let $G$ be a cyclic group of order $n \geq 2$ and $S$ be a sequence over $G$ of length $|S|=n$. If $\mathrm{h}(S)<4$ or $\mathrm{h}(S) \geq n / 2$, then $S$ has a subsequence $T$ with $\operatorname{ind}(T)=1$ and length $|T| \leq \mathrm{h}(S)$.

Theorem 1.4. Let $G$ be a cyclic group of prime order $p>24318$ and $S$ be a sequence over $G$ of length $|S|=p$. If $\mathrm{h}(S) \geq \frac{p-2}{10}$, then $S$ has a subsequence $T$ with $\operatorname{ind}(T)=1$.

In Section 2 we summarize our notations and give the proof of Theorem 1.2, In the following two sections we provide the proofs of Theorem 1.3 and of Theorem 1.4. We end the paper with a further conjecture and some open problems (see Section 5).

\section{Notations and Proof of Theorem 1.2}

Let $\mathbb{N}$ denote the set of positive integers, $\mathbb{P} \subset \mathbb{N}$ the set of prime numbers, and for rational numbers $a, b \in \mathbb{Q}$ we set $[a, b]=\{x \in \mathbb{Z} \mid a \leq x \leq b\}$. Let $G$ be an additively written abelian group and $G_{0} \subset G$ a subset. We fix the notation concerning sequences over $G_{0}$ (which is consistent with [4] and [9]). Let $\mathcal{F}\left(G_{0}\right)$ be the free abelian monoid with basis $G_{0}$. The elements of $\mathcal{F}\left(G_{0}\right)$ are called sequences over $G_{0}$. We write sequences $S \in \mathcal{F}\left(G_{0}\right)$ in the form

$$
S=g_{1} \cdot \ldots \cdot g_{l}=\prod_{g \in G} g^{\mathrm{v}_{g}(S)},
$$

where $l \in \mathbb{N}_{0}, g_{1}, \ldots, g_{l} \in G_{0}, \mathrm{v}_{g}(S) \in \mathbb{N}_{0}$ and $\mathrm{v}_{g}(S)=0$ for almost all $g \in G_{0}$. We call $|S|=l$ the length of $S, \sigma(S)=g_{1}+\ldots+g_{l}$ the sum of $S, \vee_{g}(S)$ the multiplicity of $g$ in $S$, $\operatorname{supp}(S)=\left\{g \in G \mid \mathrm{v}_{g}(S)>0\right\}$ the support of $S$, and we denote by

$$
\mathrm{h}(S)=\max \left\{\mathrm{v}_{g}(S) \mid g \in G\right\} \in[0,|S|] \quad \text { the maximum of the multiplicities of } S .
$$

For every group homomorphism $\varphi: G \rightarrow H$, we set $\varphi(S)=\varphi\left(g_{1}\right) \cdot \ldots \cdot \varphi\left(g_{l}\right) \in \mathcal{F}(H)$, and if $\varphi$ is the multiplication by some $m \in \mathbb{N}$, then we set $m S=\varphi(S)$. We say that $S$ is a zero-sum 
sequence if $\sigma(S)=0$, and it is called a minimal zero-sum sequence if $\sigma(S)=0$ but $\sum_{i \in I} g_{i} \neq 0$ for all $\emptyset \neq I \subsetneq[1, l]$. Suppose that $G$ is finite cyclic. Then a simple calculation (see [8, Lemma $5.1 .2])$ shows that

$$
\begin{aligned}
\operatorname{ind}(S) & =\min \left\{\|S\|_{h} \mid h \in G \text { with } \operatorname{supp}(S) \subset\langle h\rangle\right\} \\
& =\min \left\{\|S\|_{h} \mid h \in G \text { with }\langle\operatorname{supp}(S)\rangle=\langle h\rangle\right\} .
\end{aligned}
$$

Proof of Theorem 1.2. Assume to the contrary that $S$ has a subsequence $T$ with $\operatorname{ind}(T)=1$. Then there exists an element $h \in G$ with $\operatorname{ord}(h)=n$ such that $\|T\|_{h}=1$. We set

$$
g=j h \quad \text { and } \quad T=g^{x}\left(\frac{n}{2} g\right)^{y}\left(\left(\frac{n}{2}+1\right) g\right)^{z}\left(\left(\frac{n}{2}+2\right) g\right)^{w}
$$

where $j \in[1, n-1]$ with $\operatorname{gcd}(j, n)=1, x \in[0, n / 2-3], y \in[0,1], z \in[0, n / 2-1]$ and $w \in[0, n / 4-2]$. Then

$$
n\|T\|_{g}=(x+z+2 w)+\frac{n}{2}(y+z+w) \equiv 0 \quad(\bmod n) .
$$

Case 1. $j<\frac{n}{4}$.

Then

$$
T=(j h)^{x}\left(\frac{n}{2} h\right)^{y}\left(\left(\frac{n}{2}+j\right) h\right)^{z}\left(\left(\frac{n}{2}+2 j\right) h\right)^{w} .
$$

Since $\|T\|_{h}=1$, we infer that $y+z+w \leq 1$ which implies that $n\|T\|_{g} \leq x+\left(\frac{n}{2}+2\right) \leq$ $\frac{n}{2}-3+\frac{n}{2}+2<n$, a contradiction.

Case 2. $\frac{n}{4}<j<\frac{n}{2}$.

Then

$$
T=(j h)^{x}\left(\frac{n}{2} h\right)^{y}\left(\left(\frac{n}{2}+j\right) h\right)^{z}\left(\left(2 j-\frac{n}{2}\right) h\right)^{w} .
$$

Since $\|T\|_{h}=1$, we infer that $x \leq 3$ and $z \leq 1$ which implies that $n\|T\|_{g} \leq x+z+2 w \leq$ $3+1+2\left(\left\lfloor\frac{n}{4}\right\rfloor-2\right)<\frac{n}{2}$. Since $x+z+2 w>0$ and again by $\|T\|_{h}=1$, we derive that $x+z+2 w \equiv 0$ $\left(\bmod \frac{n}{2}\right)$, a contradiction.

Case 3. $\frac{n}{2}<j<\frac{3 n}{4}$.

Then

$$
T=(j h)^{x}\left(\frac{n}{2} h\right)^{y}\left(\left(j-\frac{n}{2}\right) h\right)^{z}\left(\left(2 j-\frac{n}{2}\right) h\right)^{w} .
$$

Since $\|T\|_{h}=1$, we infer that $x+y+w \leq 1$. We assert that

$$
x+y+w=1 \text {. }
$$

Otherwise, $x=y=w=0$ and $n\|T\|_{g}=z+\frac{n}{2} z \not \equiv 0\left(\bmod \frac{n}{2}\right)$, a contradiction to $n\|T\|_{g} \equiv 0$ $(\bmod n)$. Note that $0<x+z+2 w<n$. By (1), we have that

$$
x+z+2 w=\frac{n}{2}
$$

and

$$
y+z+w \equiv 1 \quad(\bmod 2) .
$$

By (2) and (3), we have $y+z+w \equiv z+w-y=\frac{n}{2}-1 \equiv 0(\bmod 2)$, a contradiction to (4).

Case 4. $\frac{3 n}{4}<j<n$. 
Then

$$
T=(j h)^{x}\left(\frac{n}{2} h\right)^{y}\left(\left(j-\frac{n}{2}\right) h\right)^{z}\left(\left(2 j-\frac{3 n}{2}\right) h\right)^{w} .
$$

Since $\|T\|_{h}=1$, we infer that $x \leq 1$ and $z \leq 3$ which implies that $n\|T\|_{g} \leq x+z+2 w \leq$ $1+3+2\left(\left\lfloor\frac{n}{4}\right\rfloor-2\right)<\frac{n}{2}$. Clearly, $x+z+2 w>0$. From (1), we derive a contradiction.

\section{Proof of Theorem 1.3}

We need the following two results. A simple proof of the first one can be found in 8 , Proposition 4.2.6] (for historical comments see [10]), and a proof of Lemma 3.2 is given in [13].

Lemma 3.1. Let $G$ be a finite cyclic group and $S$ be a sequence over $G$ of length $|S| \geq|G|$. Then $S$ has a zero-sum subsequence $T$ of length $|T| \in[1, \mathrm{~h}(S)]$.

Lemma 3.2. Let $G$ be a finite cyclic group and $S$ be a minimal zero-sum sequence over $G$ of length $|S| \in[1,3]$. Then $\operatorname{ind}(S)=1$.

Proof of Theorem 1.3. We set $n=|G|$ and $h=\mathrm{h}(S)$. If $h<4$, then the assertion follows from Lemmas 3.1 and 3.2. Suppose that $h \geq n / 2$. Let $g \in G$ with $\vee_{g}(S)=h$. If $\operatorname{ord}(g)<n$, then $\operatorname{ord}(g) \leq n / 2 \leq h$, and $T=g^{\operatorname{ord}(g)}$ has the required properties. If $0 \mid S$, then $T=0$ has the required properties.

Suppose that $\operatorname{ord}(g)=n$ and that $0 \nmid S$. Then we can write $S$ in the form

$$
S=g^{h}\left(b_{1} g\right) \cdot \ldots \cdot\left(b_{n-h} g\right) \quad \text { where } \quad b_{1}, \ldots, b_{n-h} \in[2, n-1] .
$$

Assume to the contrary that $S$ has no subsequence $T$ with the required properties. We continue with the following assertion.

A. For every subset $I \subset[1, n-h]$ we have $\sum_{i \in I} b_{i} \leq n-h+|I|-1$.

If $\mathbf{A}$ holds, then we apply it with $I=[1, n-h]$ and obtain that

$$
\sum_{i=1}^{n-h} b_{i} \leq 2(n-h)-1
$$

a contradiction to $b_{1}, \ldots, b_{n-h} \in[2, n-1]$. We prove $\mathbf{A}$ by induction on $|I|$. If there were an $i \in[1, n-h]$ such that $b_{i} \geq n-h+1$, then $T=g^{n-b_{i}}\left(b_{i} g\right)$ were a subsequence of $S$ with $\operatorname{ind}(T)=1$ and length $|T|=n-b_{i}+1 \leq h$, a contradiction. Let $I \subset[1, n-h]$ with $|I|=k+1 \geq 2$, say $I=[1, k+1]$, and suppose that $\mathbf{A}$ holds for all proper subsets of $I$. We set $\beta=b_{1}+\ldots+b_{k+1}$. By induction hypothesis we get $\beta-b_{i} \leq n-h+k-1$ for every $i \in[1, k+1]$, which implies that

$$
\beta=\frac{1}{k}(k \beta)=\frac{1}{k} \sum_{i=1}^{k+1}\left(\beta-b_{i}\right) \leq \frac{(k+1)(n-h+k-1)}{k} \leq n
$$

(to get the last inequality, use that $h \geq n / 2$ and $k \leq n-h-1$ ). Thus, if $\beta \geq n-h+k+1$, then $T=g^{n-\beta}\left(b_{1} g\right) \cdot \ldots \cdot\left(b_{k+1} g\right)$ is a subsequence of $S$ with $\operatorname{ind}(T)=1$ and length $|T|=$ $n-\beta+k+1 \leq h$. This is a contradiction, and thus $\mathbf{A}$ is proved. 
Note that the sequence $S$ given in Theorem 1.2 satisfies $\mathrm{h}(S)=\frac{n}{2}-1$. Thus the assumption in Theorem 1.3, that $\mathrm{h}(S) \geq \frac{n}{2}$, cannot be weakened for $n \equiv 2(\bmod 4)$.

\section{Proof of Theorem 1.4}

We fix our notations which remain valid throughout the whole section. Let $G$ be a prime cyclic group of order $|G|=p>24318, G^{\bullet}=G \backslash\{0\}$, and let $S$ be a sequence over $G^{\bullet}$ of length $|S|=p$. If $g \in G^{\bullet}, A \subset \mathbb{Z}$ and $S=\left(n_{1} g\right) \cdot \ldots \cdot\left(n_{l} g\right)$ with $n_{1}, \ldots, n_{l} \in[1, p-1]$, then we set

$$
S(A, g)=\prod_{i \in[1, l], n_{i} \in A}\left(n_{i} g\right) .
$$

For an element $g \in G^{\bullet}$, we set

$$
\Sigma_{g}(S)=\left\{p\|T\|_{g} \mid T \text { is a subsequence of } S \text { with }\|T\|_{g} \leq 1\right\},
$$

and we denote by $\mathrm{m}_{g}(S)$ the maximal $t \in[1, p]$ such that $\Sigma_{g}(T)=[1, t]$ for some subsequence $T$ of $S$. We define

$$
\mathrm{m}(S)=\max \left\{\mathrm{m}_{g}(S) \mid g \in G^{\bullet}\right\} .
$$

From now on we fix an element $g \in G^{\bullet}$ such that $\mathrm{m}_{g}(S)=\mathrm{m}(S)$.

Lemma 4.1. Let $T$ be a subsequence of $S$ such that $\Sigma_{g}(T)=[1, \mathrm{~m}(S)]$. Then $|T| \leq \mathrm{m}(S)$, and if $x \in[1, p-1]$ such that $(x g) \mid S T^{-1}$, then $x \geq \mathrm{m}(S)+2$. Furthermore, if $\mathrm{m}(S)=p$, or if there exists an $x \in[1, p-1]$ such that $(x g) \mid S T^{-1}$ and $x \geq p-\mathrm{m}(S)$, then $S$ has a subsequence with index 1.

Proof. By definition, we have $|T| \leq p\|T\|_{g}=\mathrm{m}(S)$. If there is some $x \in[1, p-1]$ with $(x g) \mid S T^{-1}$ and $x \leq \mathrm{m}(S)+1$, then $\Sigma_{g}((x g) T)=[1, \min \{p, \mathrm{~m}(S)+x\}]$, a contradiction to the maximality of $\mathrm{m}(S)$. The second part of this lemma is clear.

From now on we suppose that $S$ has no subsequence with index 1.

Let $k \geq 2$ be a positive integer, and let $F\left[\frac{1}{k}, \frac{k-1}{k}\right]$ be all irreducible fractions between $\frac{1}{k}$ and $\frac{k-1}{k}$ and with denominators in $[2, k]$, i.e.,

$$
F\left[\frac{1}{k}, \frac{k-1}{k}\right]=\left\{\frac{a}{b} \mid a \in \mathbb{N}, b \in[2, k] \text { with } \operatorname{gcd}(a, b)=1 \text { and } \frac{1}{k} \leq \frac{a}{b} \leq \frac{k-1}{k}\right\}
$$

Lemma 4.2. Let $\frac{a}{b}$ and $\frac{c}{d}$ be two adjacent fractions in $F\left[\frac{1}{k}, \frac{k-1}{k}\right]$ with $\frac{a}{b}<\frac{c}{d}$. Then we have

1. $b+d \geq k+1$.

2. $b c-a d=1$.

Proof. 1. Note that $\frac{a}{b}<\frac{a+c}{b+d}<\frac{c}{d}$. Since $\frac{a}{b}$ and $\frac{c}{d}$ are adjacent, it follows that the irreducible fraction with value $\frac{a+c}{b+d}$ is not in $F\left[\frac{1}{k}, \frac{k-1}{k}\right]$. This forces that $b+d \geq k+1$.

2. Since $\operatorname{gcd}(a, b)=1$, there are two integers $u$ and $v$ such that $b u+a v=1$. Note that $b(u+m a)+a(v-m b)=1$ holds for any integer $m$. Let $x=u+m a$ and $y=m b-v$. Then, 
$b x-a y=1$. By choosing $m$ suitably we may assume that $y \leq k$ and $y+b \geq k+1$. It follows that $y \geq k+1-b>0$ and $x>0$. From $b x-a y=1$ we get

$$
\frac{x}{y}-\frac{a}{b}=\frac{1}{b y} \text {. }
$$

If $y>1$, then $\frac{x}{y}$ is a fraction in $F\left[\frac{1}{k}, \frac{k-1}{k}\right]$. So, either $\frac{c}{d}=\frac{x}{y}$ and we are done, or $\frac{c}{d}<\frac{x}{y}$. For the latter case we have $\frac{1}{b y}=\frac{x}{y}-\frac{a}{b}=\left(\frac{x}{y}-\frac{c}{d}\right)+\left(\frac{c}{d}-\frac{a}{b}\right)=\frac{b(d x-c y)+y(c b-a d)}{b y d} \geq \frac{b+y}{b y d}$. This implies that $d \geq b+y \geq k+1$, a contradiction.

Now assume that $y=1$ and we must have $b=k$. It follows from $b x-a y=1$ that $a=k x-1$. Therefore, $x=1$ and $a=k-1$. So, $\frac{a}{b}=\frac{k-1}{k}$ is the biggest fraction in $F\left[\frac{1}{k}, \frac{k-1}{k}\right]$, a contradiction.

We set

$$
k=\left\lfloor\frac{p}{\mathrm{~m}(S)}\right\rfloor, \quad f=\left|F\left[\frac{1}{k}, \frac{k-1}{k}\right]\right|,
$$

and we arrange all fractions in $F\left[\frac{1}{k}, \frac{k-1}{k}\right]$ increasingly; so let

$$
\frac{a_{1}}{b_{1}}<\ldots<\frac{a_{f}}{b_{f}}
$$

denote the elements of $F\left[\frac{1}{k}, \frac{k-1}{k}\right]$. Furthermore, we set

$$
S_{1}=S([1, \mathrm{~m}(S)], g) \quad S_{2}=S\left(\left[\mathrm{~m}(S)+2, \frac{p-1}{b_{1}}\right], g\right)
$$

and, for every $i \in[1, f]$, we set

$$
S_{2 i+1}=S\left(\left[\frac{a_{i} p+1}{b_{i}}, \frac{a_{i} p+\mathrm{m}(S)}{b_{i}}\right], g\right) \quad \text { and } \quad S_{2 i+2}=S\left(\left[\frac{a_{i} p+\mathrm{m}(S)+1}{b_{i}}, \frac{a_{i+1} p-1}{b_{i+1}}\right], g\right) .
$$

Furthermore, for every $i \in[2, k]$, we define

$$
R_{i}=S\left(\left\{x \in[1, p] \mid \text { If } x_{i} \in[1, p] \text { with } p \mid\left(x_{i}-i x\right) \text {, then } x_{i} \in[1, \mathrm{~m}(S)] \text { and } \operatorname{gcd}\left(x_{i}, i\right)=1\right\}, g\right) .
$$

Lemma 4.3. We have $S=\prod_{j=1}^{2 f+1} S_{j}$.

Proof. This is clear by construction.

Lemma 4.4. Suppose that

$$
4 \leq \mathrm{m}(S) \leq \frac{p-3}{2} \quad \text { and } \quad \max \left\{\frac{p-\mathrm{m}(S)-2}{\mathrm{~m}(S)}, \frac{p-\mathrm{m}(S)}{\mathrm{m}(S)+1}\right\} \leq k \leq \frac{p+1}{\mathrm{~m}(S)} .
$$

1. $\left|S_{2 i+2}\right| \leq b_{i+1}-1$ for every $i \in[0, f-1]$.

2. $p=|S| \leq \mathrm{m}(S)+\sum_{i=2}^{k} \sum_{j \in[1, i-1] \text { with } \operatorname{gcd}(i, j)=1}(i-1)+\sum_{i=2}^{k}\left|R_{i}\right|$.

Proof. 1. Suppose that $i=0$. Then $S_{2}=S\left(\left[\mathrm{~m}(S)+2, \frac{p-1}{b_{1}}\right], g\right)$ and $b_{1}=k$. If $\left|S_{2}\right| \geq b_{1}=k$, then we can take a $k$-term subsequence $U$ of $S_{2}$. Note that $p-1 \geq p\|U\|_{g} \geq k(\mathrm{~m}(S)+2) \geq p-\mathrm{m}(S)$ and one can find a subsequence $V$ of $S_{1}$ such that $U V$ has index 1, a contradiction. 
Now suppose that $i \in[1, f-1]$, and assume to the contrary that $\left|S_{2 i+2}\right| \geq b_{i+1}$. We choose an arbitrary $b_{i+1}$-term subsequence $X$ of $S_{2 i+2}$, and write $b_{i} S$ in the form

$$
b_{i} S=\left(x_{1} g\right) \cdot \ldots \cdot\left(x_{p} g\right) \quad \text { with } \quad x_{1}, \ldots, x_{p} \in[1, p-1] .
$$

It follows from Lemma 4.2 that $a_{i+1} b_{i}-a_{i} b_{i+1}=1$, and so $b_{i}\left(\frac{a_{i+1} p-1}{b_{i+1}}\right)-a_{i} p=\frac{p-b_{i}}{b_{i+1}}$. Thus for every $\nu \in[1, p]$ with $\left(x_{\nu} g\right) \mid S_{2 i+2}$, we infer that $x_{\nu} \in\left[\mathrm{m}(S)+1, \frac{p-b_{i}}{b_{i+1}}\right]$ and $x_{\nu} \equiv-a_{i} p\left(\bmod b_{i}\right)$. Therefore we get, since by Lemma $4.2, b_{i}+b_{i+1} \geq k+1$,

$$
p-b_{i} \geq p\left\|b_{i} X\right\|_{g} \geq b_{i+1}(\mathrm{~m}(S)+1) \geq p-b_{i} \mathrm{~m}(S)
$$

and

$$
p\left\|b_{i} X\right\|_{g} \equiv-b_{i+1} a_{i} p=\left(1-a_{i+1} b_{i}\right) p \equiv p \quad\left(\bmod b_{i}\right) .
$$

Therefore there exists a subsequence $Y$ of $S_{1}$ such that $p\left\|b_{i}(X Y)\right\|_{g}=p$, a contradiction.

2. For every $\ell \in[2, k]$, we have $R_{\ell}=\prod_{b_{i}=\ell} S_{2 i+1}$, and hence

$$
S=S_{1} \prod_{i=0}^{f-1} S_{2 i+2} \prod_{\ell=2}^{k} R_{\ell}
$$

Now 2. follows from 1.

Lemma 4.5. Let $\ell \in \mathbb{N}_{\geq 2}$ and $S \in \mathcal{F}(\mathbb{Z})$ be a sequence of length $|S|=\ell$. Suppose that every element from $S$ is co-prime to $\ell$. Then for every $m \in \mathbb{Z}$ there exists a subsequence $S_{m}$ such that $\sigma\left(S_{m}\right) \equiv m(\bmod \ell)$. Moreover, if $m \notin \ell \mathbb{Z}$, then we get $S_{m} \neq S$.

Proof. Let $\varphi: \mathbb{Z} \rightarrow \mathbb{Z} / \ell \mathbb{Z}$ be the canonical epimorphism and $\varphi(S)=a_{1} \cdot \ldots \cdot a_{l}$. We denote by $A=\left\{a_{1}, 0\right\}+\ldots+\left\{a_{\ell-1}, 0\right\} \subset \mathbb{Z} / \ell \mathbb{Z}$ the sumset, and by $H=\operatorname{Stab}(A)$ the stabilizer of $A$. Clearly, it suffices to verify that $A=\mathbb{Z} / \ell \mathbb{Z}$. If $H$ would be a proper subgroup of $\mathbb{Z} / \ell \mathbb{Z}$, then Kneser's Theorem would imply that

$$
|A| \geq \sum_{i=1}^{\ell-1}\left|\left\{a_{i}, 0\right\}+H\right|-(\ell-2)|H|=(\ell-1) 2|H|-(\ell-2)|H| \geq \ell,
$$

whence $A=H=Z / \ell \mathbb{Z}$. Thus $H=\mathbb{Z} / \ell \mathbb{Z}$, which implies that $A=\mathbb{Z} / \ell \mathbb{Z}$, and we are done.

Lemma 4.6. Let $t, \ell \in[2, k-1]$ with $t<\ell$ and $d=\operatorname{gcd}(t, \ell)<t$, and let $u \in[2, \mathrm{~m}(S)]$. If $\frac{(t-d) p-\ell}{t \ell} \leq \mathrm{m}(S) \leq \frac{d p}{\ell}-t(u-1), \quad$ then $\quad\left|R_{t}\right|=0 \quad$ or $\quad\left|R_{\ell}\right| \leq \frac{p-\ell \mathrm{m}(S)-2 \ell+1}{u}+2 \ell-1$.

Proof. Suppose that $\left|R_{t}\right|>0$. Let $x \in[1, p-1]$ such that $(x g) \mid R_{t}$, and let $x_{\ell} \in[1, p-1]$ such that $p \mid\left(\ell x-x_{\ell}\right)$. By the definition of $R_{t}$, we get

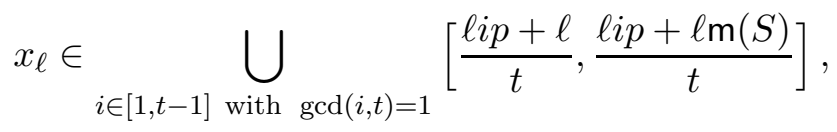

and thus,

$$
x_{\ell} \in \bigcup_{i \in[1, t-1] \text { with } d \mid i}\left[\frac{i p+\ell}{t}, \frac{i p+\ell \mathrm{m}(S)}{t}\right] \subset\left[\frac{d p+\ell}{t}, \frac{(t-d) p+\ell \mathrm{m}(S)}{t}\right] \subset[p-\ell \mathrm{m}(S), p-\ell(u-1)] .
$$

If $\left|\left(\ell R_{\ell}\right)([1, u-1], g)\right| \geq \ell$, then, by Lemma 4.5 and the definition of $R_{t}$, we may choose a subsequence $W$ of $R_{\ell}$ of length at most $\ell$ such that $(\ell W)([1, u-1], g)=\ell W$ and $x_{\ell}+p\|\ell W\|_{g} \equiv p$ 
$(\bmod \ell)$. Since $p\|\ell W\|_{g} \leq \ell(u-1)$, we have $x_{\ell}+p\|\ell W\|_{g} \in[p-\ell \mathrm{m}(S), p]$. Thus, we can construct a subsequence of $(x g) W S_{1}$ of index 1, a contradiction. Therefore,

$$
\left|\left(\ell R_{\ell}\right)([1, u-1], g)\right| \leq \ell-1 .
$$

If $\left|R_{\ell}\right|<\ell$ then we are done. Otherwise, by Lemma 4.5, we get a subsequence $R_{0}$ of $R_{\ell}$ with $p\left\|\ell R_{0}\right\|_{p} \equiv p(\bmod \ell)$ and

$$
\left|R_{0}\right| \geq\left|R_{\ell}\right|-\ell
$$

We assert that

$$
p\left\|\ell R_{0}\right\|_{p} \leq p-\ell \mathrm{m}(S)-\ell .
$$

Assume to the contrary that $p\left\|\ell R_{0}\right\|_{p} \geq p-\ell \mathrm{m}(S)$, choose $T$ to be the minimal subsequence of $R_{0}$ such that $p\|\ell T\|_{g} \geq p-\ell \mathrm{m}(S)$ and $p\|\ell T\|_{g} \equiv p(\bmod \ell)$. If $p\|\ell T\|_{g} \leq p$, then we can construct a subsequence of $T S_{1}$ with index 1, a contradiction. Now suppose that $p\|\ell T\|_{g}>p$. If $y \in[1, p-1]$ such that $(y g) \mid R_{\ell}$ and $y_{\ell} \in[1, p-1]$ such that $p \mid\left(\ell y-y_{\ell}\right)$, then $y_{\ell} \in[1, \mathrm{~m}(S)]$ and $\operatorname{gcd}\left(y_{\ell}, \ell\right)=1$. By Lemma 4.5, by dropping at most $\ell$ terms from $T$, we get a proper subsequence $\tilde{T}$ such that $p\|\ell \tilde{T}\|_{g} \geq p-\ell \mathrm{m}(S)$ and $p\|\ell \tilde{T}\|_{g} \equiv p(\bmod \ell)$, a contradiction to the minimality of $T$. Therefore, (7) holds.

By (5), we have that $p\left\|\ell R_{0}\right\|_{g} \geq(\ell-1)+u\left(\left|R_{0}\right|-\ell+1\right)$. This together with (7) gives that $\left|R_{0}\right| \leq \frac{p-\ell \mathrm{m}(S)-2 \ell+1}{u}+\ell-1$. Now the lemma follows from (6).

Lemma 4.7. Let $t \in[2, k]$, and let $1=\alpha_{1}<\alpha_{2}<\ldots$ denote all positive integers coprime to $t$. If

then

$$
\mathrm{m}(S) \leq \frac{p-2 t+w \alpha_{u+1}+2}{t+\sum_{i=2}^{u} \alpha_{i}} \text { for some } w, u \in \mathbb{N}_{0}
$$

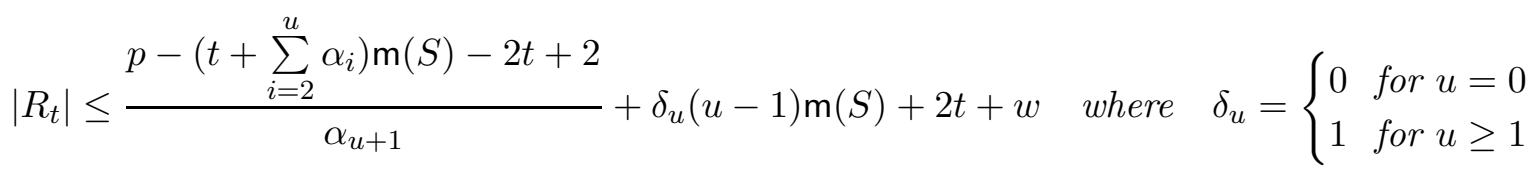

Proof. Assume to the contrary that $\left|R_{t}\right|$ is strictly larger than the above bound. Since

$$
\mathrm{m}(S) \leq \frac{p-2 t+w \alpha_{u+1}+2}{t+\sum_{i=2}^{u} \alpha_{i}}, \quad \text { it follows that }\left|R_{t}\right| \geq 2 t+1 .
$$

By Lemma 4.5, there exists a nonempty subsequence $R_{0}$ of $R_{t}$ with

$$
p\left\|t R_{0}\right\|_{g} \equiv p \quad(\bmod t) \quad \text { and } \quad\left|R_{0}\right| \geq\left|R_{t}\right|-t .
$$

Similarly to Lemma 4.6, we can prove that

$$
p\left\|t R_{0}\right\|_{g} \leq p-t \mathrm{~m}(S)-t .
$$

Note that $t R_{0}$ contains $\alpha_{1} g=g$ at most $t-2$ times, because otherwise we would get

$$
\mathrm{m}(S) \geq \mathrm{m}_{g}(t S) \geq t \mathrm{~m}_{g}(S)+t-1>\mathrm{m}_{g}(S)=\mathrm{m}(S),
$$

a contradiction. Since $\mathrm{v}_{\alpha_{i} g}(S) \leq \mathrm{h}(S) \leq \mathrm{m}(S)$ for all $i \geq 2$, it follows that

$$
p\left\|t R_{0}\right\|_{g} \geq \alpha_{1}(t-2)+\left(\sum_{i=2}^{u} \alpha_{i}\right) \mathrm{m}(S)+\alpha_{u+1}\left(\left|R_{0}\right|-(u-1) \mathrm{m}(S)-(t-2)\right) .
$$


By (9), we have $\left|R_{0}\right| \leq \frac{p-\left(t+\sum_{i=2}^{u} a_{i}\right) \mathrm{m}(S)-2 t+2}{\alpha_{u+1}}+\delta(u-1) \mathrm{m}(S)+t-2$. By (86), we derive a contradiction.

Proof of Theorem 1.4. We use all the notations which have been fixed at the beginning of this section. In particular, we assume to the contrary that there exists a sequence $S \in \mathcal{F}\left(G^{\bullet}\right)$ of length $|S|=p$ which has no subsequence with index 1. We have to derive a contradiction.

Clearly, we have $\mathrm{h}(S) \leq \mathrm{m}(S) \leq p-1$. Lemma 4.1 implies that, for every $x \in[1, p-1]$ with $(x g) \mid S T^{-1}$, we have $\mathrm{m}(S)+2 \leq x \leq p-\mathrm{m}(S)-1$. Thus it follows that

$$
\frac{p-2}{10} \leq \mathrm{h}(S) \leq \mathrm{m}(S) \leq \frac{p-3}{2} \text {. }
$$

We distinguish several cases.

Case 1. $\frac{p-2}{3} \leq \mathrm{m}(S) \leq \frac{p-3}{2}$.

With $k=2$ in Lemma 4.4, we have

$$
p \leq \mathrm{m}(S)+1+\left|R_{2}\right| .
$$

Applying Lemma 4.7 with $u=0$ and $w=6$, we infer that

$$
\left|R_{2}\right| \leq p-2 \mathrm{~m}(S)+8 \text {. }
$$

It follows that $p \leq \mathrm{m}(S)+1+\left|R_{2}\right|=\mathrm{m}(S)+1+p-2 \mathrm{~m}(S)+8<p$, a contradiction.

Case 2. $\frac{p+3}{4} \leq \mathrm{m}(S) \leq \frac{p-4}{3}$.

With $k=3$ in Lemma 4.4, we have

$$
p \leq \mathrm{m}(S)+1+2+2+\left|R_{2}\right|+\left|R_{3}\right| .
$$

Applying Lemma 4.7 with $u=1$ and $w=6$, we infer that

$$
\left|R_{2}\right| \leq \frac{p-2 \mathrm{~m}(S)+28}{3} \text { and }\left|R_{3}\right| \leq \frac{p-3 \mathrm{~m}(S)+20}{2} .
$$

It follows that

$$
p \leq \mathrm{m}(S)+5+\sum_{i=2}^{3}\left|R_{i}\right|=\mathrm{m}(S)+5+\frac{p-2 \mathrm{~m}(S)+28}{3}+\frac{p-3 \mathrm{~m}(S)+20}{2}<p,
$$

a contradiction.

Case 3. $\frac{p-2}{5} \leq \mathrm{m}(S) \leq \frac{p+1}{4}$.

With $k=4$ in Lemma 4.4, we have

$$
p \leq \mathrm{m}(S)+1+2 \cdot 2+3 \cdot 2+\left|R_{2}\right|+\left|R_{3}\right|+\left|R_{4}\right| .
$$

Applying Lemma 4.7 with $u=1$ and $w=6$, we infer that

$$
\left|R_{2}\right| \leq \frac{p-2 \mathrm{~m}(S)+28}{3}, \quad\left|R_{3}\right| \leq \frac{p-3 \mathrm{~m}(S)+20}{2} \quad \text { and } \quad\left|R_{4}\right| \leq \frac{p-4 \mathrm{~m}(S)+36}{3} .
$$

It follows that

$$
p \leq \mathrm{m}(S)+11+\frac{p-2 \mathrm{~m}(S)+28}{3}+\frac{p-3 \mathrm{~m}(S)+20}{2}+\frac{p-4 \mathrm{~m}(S)+36}{3}<p,
$$


a contradiction.

Case 4. $\frac{p-1}{6} \leq \mathrm{m}(S) \leq \frac{p-3}{5}$.

With $k=5$ in Lemma 4.4, we have

$$
p \leq \mathrm{m}(S)+27+\sum_{i=2}^{5}\left|R_{i}\right| .
$$

Applying Lemma 4.7 with $u=1$ and $w=6$, we infer that

$$
\begin{array}{ll}
\left|R_{2}\right| \leq \frac{p-2 \mathrm{~m}(S)+28}{3}, & \left|R_{3}\right| \leq \frac{p-3 \mathrm{~m}(S)+20}{2}, \\
\left|R_{4}\right| \leq \frac{p-4 \mathrm{~m}(S)+36}{3}, & \left|R_{5}\right| \leq \frac{p-5 \mathrm{~m}(S)+24}{2} .
\end{array}
$$

Applying Lemma 4.6 with $t=2, \ell=3$ and $u=12$, we obtain that either

$$
\left|R_{2}\right|=0 \quad \text { or } \quad\left|R_{3}\right| \leq \frac{p-3 \mathrm{~m}(S)+55}{12},
$$

and therefore

$\left|R_{2}\right|+\left|R_{3}\right| \leq \max \left\{\frac{p-2 \mathrm{~m}(S)+28}{3}+\frac{p-3 \mathrm{~m}(S)+55}{12}, \frac{p-3 \mathrm{~m}(S)+20}{2}\right\}=\frac{5 p-11 \mathrm{~m}(S)+167}{12}$.

Summing up we obtain that

$$
\begin{aligned}
p & \leq \mathrm{m}(S)+27+\sum_{i=2}^{5}\left|R_{i}\right|=\mathrm{m}(S)+27+\left(\left|R_{2}\right|+\left|R_{3}\right|\right)+\left|R_{4}\right|+\left|R_{5}\right| \\
& \leq \frac{5 p-11 \mathrm{~m}(S)+167}{12}+\frac{p-4 \mathrm{~m}(S)+36}{3}+\frac{p-5 \mathrm{~m}(S)+24}{2}+27<p,
\end{aligned}
$$

a contradiction.

Case 5. $\frac{p-5}{7} \leq \mathrm{m}(S) \leq \frac{p-5}{6}$.

With $k=6$ in Lemma 4.4, we have

$$
p \leq \mathrm{m}(S)+37+\sum_{i=2}^{6}\left|R_{i}\right| .
$$

Applying Lemma 4.7 with $u=2$ and $w=0$, we infer that

$$
\left|R_{2}\right| \leq \frac{p+18}{5} \text { and }\left|R_{3}\right| \leq \frac{p-\mathrm{m}(S)+20}{4} .
$$

Applying Lemma 4.7 with $u=1$ and $w=6$, we infer that

$$
\left|R_{4}\right| \leq \frac{p-4 \mathrm{~m}(S)+36}{3}, \quad\left|R_{5}\right| \leq \frac{p-5 \mathrm{~m}(S)+24}{2}, \quad \text { and } \quad\left|R_{6}\right| \leq \frac{p-6 \mathrm{~m}(S)+80}{5} .
$$

Summing up we obtain that

$$
\begin{aligned}
p & \leq \mathrm{m}(S)+37+\sum_{i=2}^{6}\left|R_{i}\right| \\
& =\mathrm{m}(S)+37+\frac{p+18}{5}+\frac{p-\mathrm{m}(S)+20}{4}+\frac{p-4 \mathrm{~m}(S)+36}{3}+\frac{p-5 \mathrm{~m}(S)+24}{2}+\frac{p-6 \mathrm{~m}(S)+80}{5} \\
& <p,
\end{aligned}
$$

a contradiction. 
Case 6. $\frac{p-2}{8} \leq \mathrm{m}(S) \leq \frac{p-3}{7}$.

With $k=7$ in Lemma 4.4, we have

$$
p \leq \mathrm{m}(S)+73+\sum_{i=2}^{7}\left|R_{i}\right| .
$$

Applying Lemma 4.7 with $u=2$ and $w=0$, we infer that

$$
\left|R_{2}\right| \leq \frac{p+18}{5} \quad \text { and } \quad\left|R_{3}\right| \leq \frac{p-\mathrm{m}(S)+20}{4}
$$

Applying Lemma 4.7 with $u=1$ and $w=6$, we infer that

$$
\begin{array}{ll}
\left|R_{4}\right| \leq \frac{p-4 \mathrm{~m}(S)+36}{3}, & \left|R_{5}\right| \leq \frac{p-5 \mathrm{~m}(S)+24}{2}, \\
\left|R_{6}\right| \leq \frac{p-6 \mathrm{~m}(S)+80}{5}, & \left|R_{7}\right| \leq \frac{p-7 \mathrm{~m}(S)+28}{2} .
\end{array}
$$

Applying Lemma 4.6, with $t=2, \ell=5$ and $u=10$, we infer that

$$
\left|R_{2}\right|+\left|R_{5}\right| \leq \max \left\{\frac{p-5 \mathrm{~m}(S)+4}{2}, \frac{p+18}{5}+\frac{p-5 \mathrm{~m}(S)-9}{10}+9\right\}=\frac{3 p-5 \mathrm{~m}(S)+117}{10} .
$$

Summing up we obtain that

$$
\begin{aligned}
p \leq \mathrm{m}(S)+ & 73+\sum_{i=2}^{7}\left|R_{i}\right|=\mathrm{m}(S)+73+\left(\left|R_{2}\right|+\left|R_{5}\right|\right)+\left|R_{3}\right|+\left|R_{4}\right|+\left|R_{6}\right|+\left|R_{7}\right| \\
\leq \mathrm{m}(S)+ & 73+\frac{3 p-5 \mathrm{~m}(S)+117}{10}+\frac{p-\mathrm{m}(S)+20}{4}+\frac{p-4 \mathrm{~m}(S)+36}{3} \\
& +\frac{p-6 \mathrm{~m}(S)+80}{5}+\frac{p-7 \mathrm{~m}(S)+28}{2}<p,
\end{aligned}
$$

a contradiction.

Case 7. $\frac{p-2}{9} \leq \mathrm{m}(S) \leq \frac{p-3}{8}$.

With $k=8$ in Lemma 4.4, we have

$$
p \leq \mathrm{m}(S)+111+\sum_{i=2}^{8}\left|R_{i}\right| .
$$

Applying Lemma 4.7 with $u=2$ and $w=0$, we infer that

$$
\begin{aligned}
& \left|R_{2}\right| \leq \frac{p+18}{5}, \quad\left|R_{3}\right| \leq \frac{p-\mathrm{m}(S)+20}{4}, \\
& \left|R_{4}\right| \leq \frac{p-2 \mathrm{~m}(S)+34}{5}, \quad\left|R_{5}\right| \leq \frac{p-4 \mathrm{~m}(S)+22}{3} .
\end{aligned}
$$

Applying Lemma 4.7 with $u=1$ and $w=6$, we infer that

$$
\left|R_{6}\right| \leq \frac{p-6 \mathrm{~m}(S)+80}{5}, \quad\left|R_{7}\right| \leq \frac{p-7 \mathrm{~m}(S)+28}{2} \quad \text { and } \quad\left|R_{8}\right| \leq \frac{p-8 \mathrm{~m}(S)+52}{3} .
$$

Applying Lemma 4.6 with $t=2, \ell \in\{5,7\}$ and $u=20$, we can prove that either

$$
\left|R_{2}\right|=0 \quad \text { or } \quad\left|R_{i}\right| \leq \frac{p-i \mathrm{~m}(S)-2 i+1}{20}+2 i-1 \quad \text { for } \quad i \in\{5,7\}
$$


and therefore

$$
\begin{aligned}
\left|R_{2}\right|+\left|R_{5}\right|+\left|R_{7}\right| \leq & \max \left\{\frac{p-4 \mathrm{~m}(S)+22}{3}+\frac{p-7 \mathrm{~m}(S)+28}{2},\right. \\
& \left.\frac{p-\mathrm{m}(S)+20}{4}+\frac{p-5 \mathrm{~m}(S)-9}{20}+9+\frac{p-7 \mathrm{~m}(S)-13}{20}+13\right\} \\
= & \frac{5 p-29 \mathrm{~m}(S)+128}{6} .
\end{aligned}
$$

Applying Lemma 4.6 with $t=4, \ell=6$ and $u=10$, we obtain that either

$$
\left|R_{4}\right|=0 \quad \text { or } \quad\left|R_{6}\right| \leq \frac{p-6 \mathrm{~m}(S)-11}{10}+11
$$

and therefore

$\left|R_{4}\right|+\left|R_{6}\right| \leq \max \left\{\frac{p-2 \mathrm{~m}(S)+34}{5}+\frac{p-6 \mathrm{~m}(S)-11}{10}+11, \frac{p-6 \mathrm{~m}(S)+80}{5}\right\}=\frac{3 p-10 \mathrm{~m}(S)+167}{10}$.

Summing up we obtain that

$$
\begin{aligned}
p & \leq \mathrm{m}(S)+111+\sum_{i=2}^{8}\left|R_{i}\right|=\mathrm{m}(S)+111+\left(\left|R_{2}\right|+\left|R_{5}\right|+\left|R_{7}\right|\right)+\left(\left|R_{4}\right|+\left|R_{6}\right|\right)+\left|R_{3}\right|+\left|R_{8}\right| \\
& \leq \mathrm{m}(S)+111+\frac{5 p-29 \mathrm{~m}(S)+128}{6}+\frac{3 p-10 \mathrm{~m}(S)+167}{10}+\frac{p-\mathrm{m}(S)+20}{4}+\frac{p-8 \mathrm{~m}(S)+52}{3} \\
& <p,
\end{aligned}
$$

a contradiction.

Case 8. $\frac{p-2}{10} \leq \mathrm{m}(S) \leq \frac{p-4}{9}$.

With $k=9$ in Lemma 4.4, we have

$$
p \leq \mathrm{m}(S)+159+\sum_{i=2}^{9}\left|R_{i}\right| .
$$

Applying Lemma 4.7 with $u=2$ and $w=0$, we infer that

$$
\begin{aligned}
\left|R_{2}\right| \leq \frac{p+18}{5}, & \left|R_{3}\right| \leq \frac{p-\mathrm{m}(S)+20}{4}, \\
\left|R_{4}\right| \leq \frac{p-2 \mathrm{~m}(S)+34}{5}, & \left|R_{5}\right| \leq \frac{p-4 \mathrm{~m}(S)+22}{3} .
\end{aligned}
$$

Applying 4.7 with $u=1$ and $w=6$, we infer that

$$
\begin{array}{ll}
\left|R_{6}\right| \leq \frac{p-6 \mathrm{~m}(S)+80}{5}, & \left|R_{7}\right| \leq \frac{p-7 \mathrm{~m}(S)+28}{2}, \\
\left|R_{8}\right| \leq \frac{p-8 \mathrm{~m}(S)+52}{3}, & \left|R_{9}\right| \leq \frac{p-9 \mathrm{~m}(S)+32}{2} .
\end{array}
$$

Applying Lemma 4.6 with $t=2, \ell \in\{5,7\}$ and $u=10$, we obtain that either

$$
\left|R_{2}\right|=0 \quad \text { or } \quad\left|R_{i}\right| \leq \frac{p-i \mathrm{~m}(S)-2 i+1}{10}+2 i-1 \quad \text { for } \quad i \in\{5,7\},
$$

and therefore

$$
\begin{aligned}
\left|R_{2}\right|+\left|R_{5}\right|+\left|R_{7}\right| & \leq \max \left\{\frac{p-4 \mathrm{~m}(S)+22}{3}+\frac{p-7 \mathrm{~m}(S)+28}{2},\right. \\
& \left.\frac{p+18}{5}+\frac{p-5 \mathrm{~m}(S)-9}{10}+9+\frac{p-7 \mathrm{~m}(S)-13}{10}+13\right\}=\frac{5 p-29 \mathrm{~m}(S)+128}{6} .
\end{aligned}
$$


Applying Lemma 4.6 with $t=3, \ell=8$ and $u=5$, we obtain that either

$$
\left|R_{3}\right|=0 \quad \text { or } \quad\left|R_{8}\right| \leq \frac{p-8 \mathrm{~m}(S)-15}{8}+15
$$

and therefore

$\left|R_{3}\right|+\left|R_{8}\right| \leq \max \left\{\frac{p-\mathrm{m}(S)+20}{4}+\frac{p-8 \mathrm{~m}(S)-15}{8}+15, \frac{p-8 \mathrm{~m}(S)+52}{3}\right\}=\frac{3 p-10 \mathrm{~m}(S)}{8}+20$.

Summing up we obtain that

$$
\begin{aligned}
p \leq \mathrm{m}(S)+ & 159+\sum_{i=2}^{9}\left|R_{i}\right|=M+159+\left(\left|R_{2}\right|+\left|R_{5}\right|+\left|R_{7}\right|\right)+\left(\left|R_{3}\right|+\left|R_{8}\right|\right)+\left|R_{4}\right|+\left|R_{6}\right|+\left|R_{9}\right| \\
\leq \mathrm{m}(S)+ & 159+\frac{5 p-29 \mathrm{~m}(S)+128}{6}+\left(\frac{3 p-10 \mathrm{~m}(S)}{8}+20\right) \\
& +\frac{p-2 \mathrm{~m}(S)+34}{5}+\frac{p-6 \mathrm{~m}(S)+80}{5}+\frac{p-9 \mathrm{~m}(S)+32}{2}<p,
\end{aligned}
$$

a contradiction.

\section{A Conjecture and Open Problems}

In spite of Theorem 1.2 and in view of Lemma 3.1, we formulate a conjecture which sharpens the original Lemke-Kleitman Conjecture for prime cyclic groups.

Conjecture 5.1. Let $G$ be a cyclic group of prime order and $S$ be a sequence over $G$ of length $|S|=|G|$. Then $S$ has a subsequence $T$ with ind $(T)=1$ and length $|T| \in[1, \mathrm{~h}(S)]$.

Let $G$ be a cyclic group of order $n \geq 2$. We denote by

- $\mathrm{t}(n)$ the smallest integer $\ell \in \mathbb{N}$ such that every sequence $S$ over $G$ of length $|S| \geq \ell$ has a subsequence $T$ with $\operatorname{ind}(T)=1$.

- T(n) the smallest integer $\ell \in \mathbb{N}$ such that every squarefree sequence $S$ over $G$ of length $|S| \geq \ell$ has a subsequence $T$ with $\operatorname{ind}(T)=1$.

By Theorem 1.2. it follows that $\mathrm{t}(n) \geq n+\left\lfloor\frac{n}{4}\right\rfloor-4$ for $n=4 k+2 \geq 22$.

Open Problem. Determine $\mathrm{t}(n)$ and $\mathrm{T}(n)$ for all $n \geq 2$.

\section{REFERENCES}

[1] S.T. Chapman, M. Freeze, and W.W. Smith, Minimal zero sequences and the strong Davenport constant, Discrete Math. 203 (1999), 271 - 277.

[2] S.T. Chapman and W.W. Smith, A characterization of minimal zero-sequences of index one in finite cyclic groups, Integers 5(1) (2005), Paper A27, 5p.

[3] W. Gao, Zero sums in finite cyclic groups, Integers 0 (2000), Paper A14, 9p.

[4] W. Gao and A. Geroldinger, Zero-sum problems in finite abelian groups: a survey, Expo. Math. 24 (2006), $337-369$.

[5] On products of $k$ atoms, Monatsh. Math. 156 (2009), $141-157$.

[6] A. Geroldinger, On non-unique factorizations into irreducible elements. II, Number Theory, Vol. II Budapest 1987, Colloquia Mathematica Societatis Janos Bolyai, vol. 51, North Holland, 1990, pp. 723 - 757. 
[7] - On a conjecture of Kleitman and Lemke, J. Number Theory 44 (1993), 60 - 65.

[8] A. Geroldinger, Additive group theory and non-unique factorizations, Combinatorial Number Theory and Additive Group Theory (A. Geroldinger and I. Ruzsa, eds.), Advanced Courses in Mathematics CRM Barcelona, Birkhäuser, 2009, pp. $1-86$.

[9] A. Geroldinger and F. Halter-Koch, Non-Unique Factorizations. Algebraic, Combinatorial and Analytic Theory, Pure and Applied Mathematics, vol. 278, Chapman \& Hall/CRC, 2006.

[10] Y. ould Hamidoune, A weighted generalization of Gao's $n+D-1$ theorem, Comb. Probab. Comput. 17 (2008), $793-798$.

[11] P. Lemke and D. Kleitman, An addition theorem on the integers modulo n, J. Number Theory 31 (1989), $335-345$.

[12] Y. Li, C. Plyley, P. Yuan, and X. Zeng, Minimal zero-sum sequences of length four over finite cyclic groups, J. Number Theory 130 (2010), 2033 - 2048.

[13] V. Ponomarenko, Minimal zero sequences of finite cyclic groups, Integers 4 (2004), Paper A24, 6p.

[14] S. Savchev and F. Chen, Long zero-free sequences in finite cyclic groups, Discrete Math. 307 (2007), 2671 2679.

[15] Xingwu Xia and Pingzhi Yuan, Indexes of insplitable minimal zero-sum sequences of length $l\left(C_{n}\right)-1$, Discrete Math. 310 (2010), 1127 - 1133.

[16] P. Yuan, On the index of minimal zero-sum sequences over finite cyclic groups, J. Comb. Theory, Ser. A 114 (2007), 1545 - 1551.

Center for Combinatorics, LPMC-TJKLC, Nankai University, Tianjin 300071, P.R. China

E-mail address: wdgao1963@yahoo.com.cn

Department of Mathematics, Brock University, St. Catharines, Ontario, Canada L2S 3A1

E-mail address: yli@brocku.ca

Center for Combinatorics, LPMC-TJKLC, Nankai University, Tianjin 300071, P.R. China

E-mail address: jtpeng1982@yahoo.com.cn

Department of Mathematics, Brock University, St. Catharines, Ontario, Canada L2S 3A1

E-mail address: cp07rp@brocku.ca

Center for Combinatorics, LPMC-TJKLC, Nankai University, Tianjin 300071, P.R. China

E-mail address: gqwang1979@yahoo.com.cn 